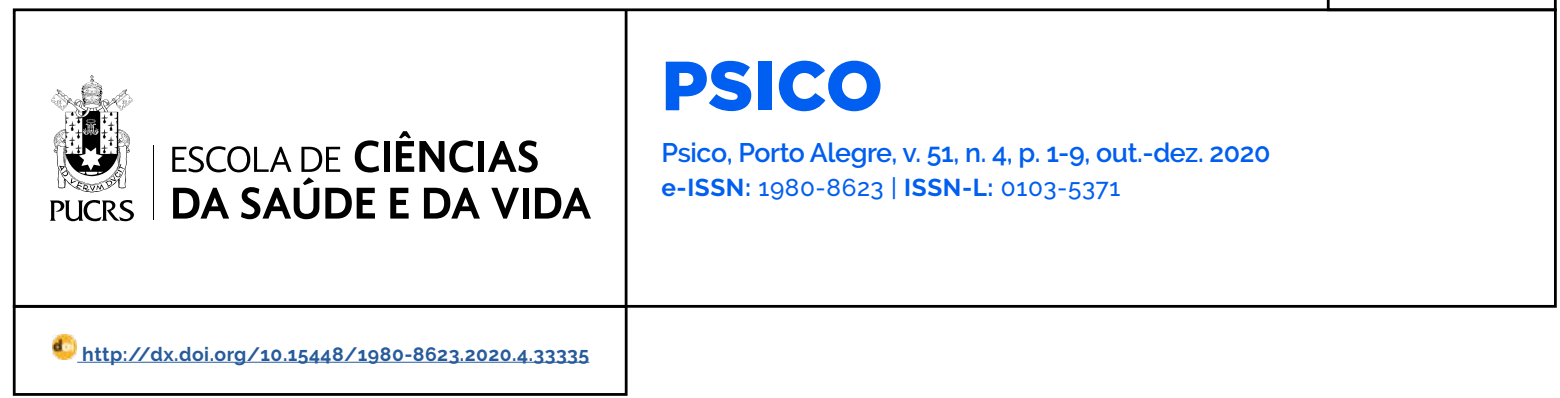

ARTIGO

\title{
Evidências de validade do "Cuestionario para la Evaluacion del Sindrome de Quemarse por el Tabajo" em profissionais da saúde
}

Evidences of validity of "Cuestionario para la Evaluacion del Sindrome de Quemarse por el Tabajo" in health professionals

Evidencias de validez del "Cuestionario para la Evaluacion del Sindrome de Quemarse por el Tabajo" en profesionales de salud

\section{Germano Gabriel Lima}

\section{Esteves $^{1}$}

orcid.org/0000-0002-1851-4603

germanoesteves@unirv.edu.br

\section{Ana Adelaide Martins \\ Leão ${ }^{2}$}

orcid.org/0000-0003-0951-8036

anaamleao@gmail.com

\section{Esther de Oliveira}

Alves $^{2}$

orcid.org/0000-0002-2662-8176

estheralves17@gmail.com

Recebido em: 13 fev. 2019

Aprovado em: 14 set. 2020.

Publicado em: 15 mar. 2021.
Resumo: A sindrome de Burnout é uma das principais causas de afastamento do trabalho, sendo necessários instrumentos psicometricamente adequados em diferentes populações. Assim, objetivou-se reunir evidências de validade de construto da versão brasileira do "Cuestionario para la Evaluación del Síndrome de Quemarse por el Trabajo" em profissionais da saúde brasileiros. Participaram 322 profissionais da área da saúde, com idade entre 21 a 62 anos (Média = 37.56: $\mathrm{DP}=10,09$ ), sendo $66,1 \%$ mulheres, de instituições públicas e privadas. Foram respondidos: (i) Questionário sociodemográfico; (ii) "Cuestionario para la Evaluación del Sindrome de Quemarse por el Trabajo" (CESQT); (iii) Escala de Estresse no Trabalho (Job Stress Scale - JSS). A análise fatorial exploratória indicou uma estrutura de quatro fatores e a confiabilidade (alfa de Cronbach) para cada uma das dimensões apresentou-se adequada. Também são apresentadas evidências de validade convergente com os fatores da JSS. Os resultados sugerem que o CESQT apresenta boas propriedades psicométricas.

Palavras-chaves: estresse ocupacional, saúde ocupacional, Burnout, validade do teste, precisão do teste

Abstract: The Burnout syndrome is one of the main causes of withdrawal from the work, requiring psychometrically adequate instruments in different populations. Therefore, the objective was to gather evidence of constructive validity from the Brazilian version of the "Cuestionario para la Evaluación del Sindrome de Quemarse por el Trabajo" in brazilians health professionals. A total of 322 health professionals, aged between 21 and 62 participated ( $M=37,56$; SD $=10,09$ ) being $66.1 \%$ women, from public and private institutions. The answered: (i) Sociodemographic questionnaire; (ii) "'Cuestionario para la Evaluación del Sindrome de Quemarse por el Trabajo"" (CESQT); (iii) Job Stress Scale (JSS). The exploratory factorial analysis indicated a four factors structure and the reliability (Cronbach's alpha) for each one of the dimensions was adequate. Evidence of convergent validity with JSS factors was also presented. The results suggest that the CESQT has good psychometric properties.

Keywords: occupational stress, occupational health, Burnout, test validity, test reliability

Resumen: El síndrome de Burnout es la mayor causa de la ausencia del trabajo, que requiere instrumentos psicométricos adecuados en diferentes poblaciones. Así, se objetivó reunir evidencias de validez de constructo de la versión brasileña del "Cuestionario para la evaluación del Sindrome de Quemarse por el Trabajo" en profesionales de la salud brasileños. Participó en 322 profesionales de la salud, edad 21-62 años ( $M=37.56, D E=10,09)$, y $66,1 \%$ de mujeres, instituciones públicas y privadas. Fueron contestadas: (i) cuestionario sociodemográfico; (ii) "Cuestionario para la evaluación del Sindrome de Quemarse por el Trabajo" (CESQT); (iii) Escala de estrés en el trabajo (Job Stress Scale - JSS). El análisis factorial exploratorio 
se indica una estructura de cuatro factores y fiabilidad (alfa de Cronbach) para cada una de las dimensiones tenía que ser apropiado. También presentan evidencia de validez convergente con los factores.

Palabras clave: estres ocupacional, salud ocupacional, Burnout, validacion de test, precisión de test

Burnout é uma sindrome que ocorre em resposta aos estressores crônicos vivenciados no trabalho que, em 2002, foi incluida na relação de doenças ocupacionais pelo Ministério da Saúde, sendo considerada um transtorno mental e do comportamento relacionados ao trabalho (Koga, et. al., 2015; (Silva, Barbosa, Silva, \& Patrício, 2015). Atualmente, Burnout é um dos problemas psicossociais presentes no trabalho mais importantes da sociedade por conta da grande quantidade de profissionais que são acometidos (Carlotto, 2010). Esse quadro ocorre devido aos sintomas físicos, psíquicos e comportamentais, como fadiga constante, cefaleia e alterações de memória (Benevides-Pereira, 2014), que impossibilitam o individuo de exercer suas atividades, levando-o a busca por afastar-se de sua função (Batista, Carlotto, Coutinho, \& Augusto, 2011). No Brasil, em 2019, o Ministério da Previdência Social reportou 145.299 casos de individuos afastados do trabalho, sendo 455 deles afastados com o diagnóstico de Burnout (ou Esgotamento, CID-10 Z73.0) (Ministério da Saúde, 2020).

Diante desse cenário, o principal instrumento utilizado nas pesquisas sobre Burnout tem sido o Inventário de Burnout Maslach (Maslach Burnout Inventory - MBI), que é composto por 22 itens divididos em três fatores: exaustão emocional, definido pelo esgotamento dos recursos emocionais ( 9 itens); despersonalização, descrito como o distanciamento, insensibilidade do sujeito ao lidar com terceiros (cinco itens); realização no trabalho, caracterizado pela propensão em se autoavaliar de forma negativa (oito itens) (Maslach, Schaufeli, \& Leiter, 2001; Trindade, Lautert, Beck, Amestoy, \& Pires, 2010). Nesse contexto, o MBI já foi utilizado no estudo de diversas populações, como professores, bancários, médicos, enfermeiros (Coelho, Souza, Cerqueira, Esteves, \& Barros, 2018; Ebling \& Carlotto, 2012; Koga, et. al., 2015; Moreira, Souza, \& Yamaguchi, 2018). No entanto,
Kristensen, Borritz, Villadsen, \& Christensen (2005) reportam a relação de um estudo piloto onde a adequação cultural do $\mathrm{MBI}$ foi testada em cerca de 70 trabalhadores de recursos humanos, indicando que a tradução de instrumentos de uma cultura, com ênfase aos construidos nos Estados Unidos da América (EUA), é prejudicada pelo foco excessivo dos itens em aspectos técnicos, deixando de lado questões de diferenças culturas, de gênero e socioeconômicas. Para além disso, todas as versões do MBI são distribuídas por uma editora comercial, o que limita a sua utilização por grande parte dos pesquisadores. Diante desse quadro de limitações culturais e práticas, outros instrumentos surgem como alternativa para avaliar Burnout, como é o caso do Cuestionario para la Evaluación del Sindrome de Quemarse por el Trabajo (CESQT), indicado como o segundo instrumento mais utilizado para avaliação do Burnout na América Latina até 2010 (Bambula \& Gómez, 2016; Gil-Monte, 2005; GilMonte, Carlotto, \& Câmara, 2010).

O CESQT, desenvolvido por Gil-Monte (2005), compreende o Burnout_como uma resposta ao estresse laboral crônico e teve seus parâmetros psicométricos evidenciados em uma amostra de cuidadores de crianças com necessidades especiais, demonstrando uma estrutura fatorial composta por quatro dimensões, a saber: (i) ilusão pelo trabalho $(\alpha=0,72)$, compreendido como as expectativas elaboradas pelo indivíduo em atingir as metas laborais, sendo essas metas fonte de realização pessoal e profissional; (ii) desgaste psíquico $(\alpha=0,72)$, entendido como o esgotamento emocional e físico do indivíduo em função das atividades exercidas no trabalho; (iii) indolência $(\alpha=0,72)$, caracterizada por atitudes negativas, indiferentes e cínicas direcionadas aos individuos que compõem o ambiente laboral; e (iv) culpa $(\alpha=0,72)$, definido como o surgimento de sentimento de culpa em função das atitudes negativas, indiferentes e cínicas direcionadas aos individuos que compõem o ambiente laboral. Nesse Contexto, o Burnout se inicia a medida em que o indivíduo não consegue se sentir realizado com o trabalho (baixa ilusão pelo trabalho) e se sente exausto emocionalmente e fisicamente 
(alto desgaste psíquico), acarretando atitudes negativas e insensiveis direcionadas ao ambiente laborais (alta indolência), o que, posteriormente, acaba por gerar o sentimento de culpa nesses individuos (Gil-Monte et al.,2010). Assim, O CESQT avalia o Burnout por meio de 20 itens, respondidos seguindo uma escala tipo Likert de quatro pontos (o "nunca" a 4 "todos os dias") (Costa, Gil-Monte, Possobon, \& Ambrosano, 2013; Gil-Monte, Carlotto, \& Câmara, 2010).

O CESQT já apresentou evidências de adequação psicométrica em diversos contextos, como em uma amostra de 110 médicos mexicanos, em que apresentou uma estrutura composta de quatro fatores: ilusão pelo trabalho $(\alpha=0,72)$, desgaste psíquico $(\alpha=0,86)$, indolência $(\alpha=0,75)$ e culpa $(\alpha=0,79)$, e bons índices de consistência interna (Gil-Monte \& Zúñiga-Caballero, 2010). Nesse sentido, em uma amostra de 695 profissionais da saúde italianos, a mesma estrutura composta por quatro fatores, ilusão pelo trabalho $(\alpha=0,90)$, desgaste psiquico $(\alpha=0,85)$, indolência $(\alpha=0,76)$ e culpa $(\alpha=0,85)$, e bons indices de consistência interna (Gil-Monte, Viotti, \& Converso, 2017). Ademais, uma metanálise realizada com 12 estudo sobre as propriedades psicométricas do CESQT indicou que sua estrutura fatorial se apresenta adequada para avaliação do Burnout (Gómez, Rivera, Tiznado, \& Grisales, 2018).

No Brasil, a adaptação do instrumento foi realizada por Gil-Monte et al. (2010) que, posteriormente, nesse mesmo estudo apresentou evidências de validade de construto, por meio de Análise Fatorial Exploratória (AFE) e Análise Fatorial Confirmatória (AFC), em uma amostra de 714 professores. Assim, por meio da AFE encontrou-se uma estrutura composta por quatro fatores: ilusão pelo trabalho $(\alpha=0,83)$, desgaste psíquico $(\alpha=0,80)$, indolência $(\alpha=0,80)$ e culpa $(\alpha=0,80$ ), e bons indices de consistência interna. Corroborando com essa estrutura a AFC indicou bons índices de ajuste para o modelo composto por quatro fatores $(\chi 2=605,86, p=0,000 ; g l=164$; $\chi 2 / \mathrm{gl}=3,69 ; \mathrm{GFI}=0,92 ; \mathrm{AGFI}=0,90 ; \mathrm{RMSEA}=0,062$; $\mathrm{NNFI}=0,91 ; \mathrm{CFI}=0.92$ ) (Gil-Monte et al., 2010). O CESQT é particularmente útil por possibilitar a composição de dois perfis de Burnout: um composto por comportamentos associados ao estresse laboral, mas não incapacitante, e caracterizado por escores baixos na dimensão ilusão pelo trabalho e culpa e escores elevados de desgaste psiquico e indolência (Perfil 1); e um segundo perfil de Burnout que indica um quadro mais grave, sendo diferenciado do anterior por elevados escores na dimensão de culpa (Perfil 2) (Gil-Monte, 2005)

Desse modo, desde a sua adaptação para o contexto brasileiro, o CESQT tem sido empregado por diferentes pesquisas em diferentes amostras, como em uma amostra de 199 gestores municipais de saúde, em que identificou 13\% dos gestores apresentavam algum nivel de Burnout (Poletto et al., 2016). Já em outro estudo realizado em uma amostra de 217 profissionais da saúde da atenção primária, constatou que $29 \%$ desses profissionais apresentaram algum nivel de Burnout (Silveira, Câmara, \& Amazarray, 2014). Além disso, o CESQT também tem sido empregado com o intuito de compreender a relação acerca do Burnout e seus preditores, como o estresse e a fadiga (Silva, Barbosa, Silva, \& Patrício, 2015).

Assim, dentre as populações investigadas estão docentes, profissionais de enfermagem, médicos e estudantes (Cardoso, Baptista, Sousa, \& Goulart, 2017). No entanto, nenhum estudo apresentou evidências de validade do CESQT em amostra de profissionais da saúde, no contexto brasileiro. Desse modo, sabendo-se que as evidências de validade são intimamente relacionadas ao contexto (AERA, APA, \& NCME, 2014), o objetivo da presente pesquisa foi reunir evidências de validade de construto (fatorial e convergente) da versão brasileira do "Cuestionario para la Evaluación del Síndrome de Quemarse por el Trabajo" em profissionais da saúde brasileiros.

\section{Método}

\section{Participantes}

Contou-se com uma amostra não probabilística, isto é, de conveniência, tendo a participação de profissionais de saúde que, convidados, aceitaram 
colaborar voluntariamente. No total, participaram 322 profissionais atuantes na área da saúde (técnicos de enfermagem (38,6\%; $f=76)$, médicos ( $9,6 \% ; f=13$ ), enfermeiros (14,7\%; $f=29$ ), odontólogos $(6,0 \% ; f=12)$, fisioterapeutas ( $14,7 \% ; f=29$ ) de diversas instituições, de diversos estados (GO, AL, BA, DF, PE e MG), sendo a maioria do estado de GO (54,6\%; $f=176$ ), com ensino superior completo (34,7\%; $f=112$ ), do sexo feminino (66,1\%; $f=213$ ), com idade média de 21 a 60 anos ( $m=37,56$; DP=10,09).

\section{Instrumentos}

Os participantes responderam aos seguintes instrumentos:

(a) Questionário sociodemográfico: constituído por dados como: cidade e estado em que trabalha, sexo, idade, escolaridade e cargo que ocupa;

(b) Cuestionario para la Evaluación del Síndrome de por el Trabajo - CESQT: elaborado por GilMonte (2005) e adaptado para o contexto brasileiro por Gil-Monte et al. (2010). Os participantes responderam de acordo com uma escala variando entre o "Nunca" e 4 "Muito Frequentemente: todos os dias". O inventário é constituido por quatro dimensões: (i) ilusão pelo trabalho; (ii) desgaste psiquico; (iii) indolência; e (iv) culpa;

(c) Escala de Estresse no Trabalho (Job Stress Scale - JSS): originária do trabalho feito por Robert Karasek (1990), formada por 49 perguntas e, posteriormente, apresentada em sua versão reduzida por Theorell (1996) a versão reduzida foi validada por Alves et. al. (2004) para o contexto brasileiro, sendo o instrumento adotado nessa pesquisa. O instrumento contém 17 itens respondidos em uma escala variando de 1 frequentemente - até 4 - nunca/quase nunca -, e divididos entre três fatores: demanda ( $\alpha=$ 0.72), comporta por cinco itens (ex: "01. Com que frequência você tem que fazer suas tarefas de trabalho com muita rapidez?"); controle ( $\alpha=0.63$ ), composto por 6 itens (Ex: "10. Você pode escolher como fazer o seu trabalho? "); e apoio social ( $\alpha=$ 0.86), composto por seis itens ("Ex: 16. Eu gosto de trabalhar com meus colegas").

\section{Procedimentos}

Inicialmente o projeto foi submetido ao Comitê de Ética em Pesquisa (CEP) da Universidade de Rio Verde - Goiás, tendo sido aprovado (CAAE: 65209617.4.0000.5077), a aplicação foi realizada por meio do questionário eletrônico divulgado em redes sociais em grupos exclusivos de profissionais da saúde. Inicialmente os participantes foram esclarecidos do caráter voluntário e sigiloso, bem como o respeito às diretrizes éticas que regem a pesquisa com seres humanos e, após, obteve-se o consentimento livre e esclarecido de todos os participantes que se voluntariaram. O tempo de coleta dos dados foi de aproximadamente 20 a 25 minutos.

\section{Análise de dados}

Inicialmente, foram realizadas, por meio do Statistical Package for the Social Sciences (SPSS) versão 24, estatísticas descritivas (média e desvio-padrão) com as respostas do questionário sociodemográfico no intuito de apresentar um perfil amostral e dos instrumentos para apresentar os escores médios dos participantes nas dimensões.

Posteriormente, por meio do programa Factor 10.8.02, foi verificado se os dados satisfaziam os pressupostos da Análise Fatorial Exploratória (AFE). Assim, como os dados foram coletados via questionário eletrônico e houve a obrigatoriedade de resposta para cada item (caso o respondente não marcasse um ponto na escala, o questionário não prosseguia para página seguinte) não houve casos ausentes. Assim, com os itens das escalas foram realizadas: análises de normalidade por meio da Skewness e Curtose de cada item e realizados testes Kolmogorov-Smirnov, os resultados indicaram a normalidade das variáveis. Também foi verificado a linearidade e não foi verificada a presença de outliers. Por fim, foi verificada a multicolinearidade por meio da matriz de correlações.

Após, os dados foram submetidos à Análise Fatorial Exploratória (AFE) no programa Factor 10.8.02, com método Unweighted Least Squares (ULS) e rotação direct oblimin. Para definição do número de fatores, utilizou-se a Análise Paralela (AP) de Horn (1965). Para análise de confiabilidade 
dos fatores foi utilizado o coeficiente alfa de Cronbach ( $\alpha$ ), por meio do software SPSS (21). Além disso, foram realizadas correlações $r$ de Pearson entre os fatores do CESQT e a JSS no intuito de reunir evidências de validade convergente.

\section{Resultados}

Inicialmente, na Tabela 1, são apresentados os escores médios, por dimensão, do CESQT no intuito de caracterizar a amostra pesquisada. Desse modo, observou-se que, adotando o ponto médio da escala como referência, a amostra foi caracterizada por escores acima do ponto médio da escala em ilusão pelo trabalho e desgaste emocional e baixos escores em indolência e culpa.

Tabela 1 - Estatísticas descritivas para as dimensões do Cuestionario para la Evaluacióndel Síndrome de por el Trabajo - CESQT

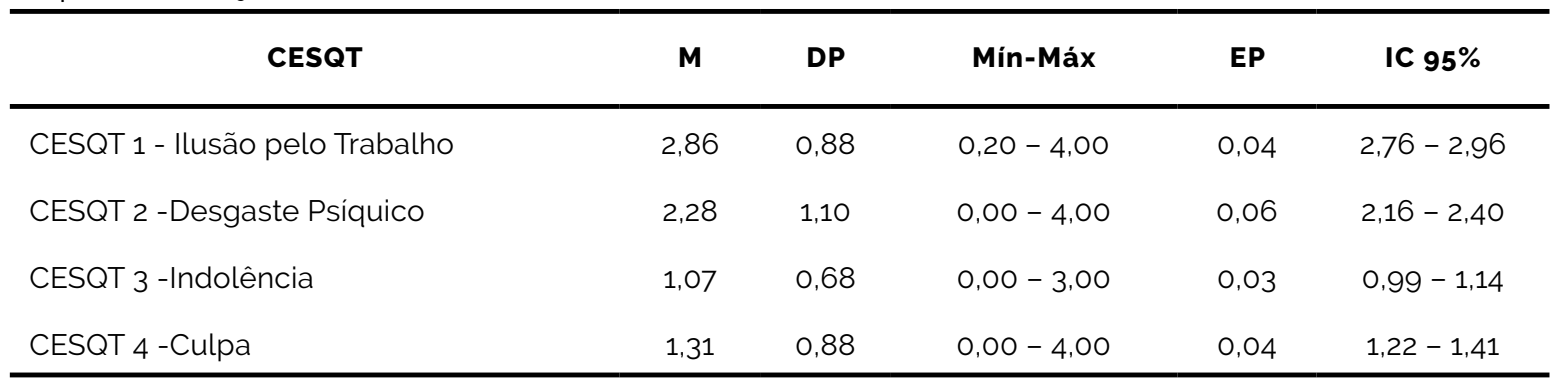

\section{Análise fatorial exploratória}

Posteriormente, buscou-se verificar a adequação dos dados à análise fatorial por meio dos testes Kaiser-Meyer-Olkin (0,87), que foi considerado aceitável, e o teste de esfericidade de Bartlett $\chi 2(190)=2719,0 ; p<0,001$, que também indicou a adequação dos dados a AFE. Para identificação do número de fatores a se extrair utilizou-se o AP de Horn, que indicou um modelo de quatro fatores, corroborando com a estrutura teórica proposta para o CESQT, ver Tabela 2.

* número de fatores que podem ser considerados

Desse modo, foi realizada uma AFE no programa Factor 10.8.02, com método Unweighted
Tabela 2 - Análise paralela de Horn

\begin{tabular}{cc}
\hline Real-data eigenvalues & Random eigenvalues \\
\hline $6.362^{*}$ & 1.470 \\
$2.700^{*}$ & 1.383 \\
$1.513^{*}$ & 1.316 \\
$1.211^{*}$ & 1.150 \\
1.013 & 1.031 \\
\hline
\end{tabular}

Least Squares (ULS) e rotação direct oblimin, fixando a extração de quatro fatores. Ver Tabela 3.

Tabela 3 - Análise fatorial para o CQUEST em profissionais da saúde

\section{Fatores}

Itens

$\begin{array}{llll}F_{1} & F_{2} & F_{3} & F_{4}\end{array}$

01. O meu trabalho representa para mim um desafio estimulante.

05. Vejo o meu trabalho como uma fonte de realização pessoal.

0.74

10. Penso que o meu trabalho me dá coisas positivas.

15. O meu trabalho me é gratificante.

0.76

19. Sinto-me encantado(a) pelo meu trabalho 


\begin{tabular}{|c|c|c|c|c|}
\hline \multirow{2}{*}{ Itens } & \multicolumn{4}{|c|}{ Fatores } \\
\hline & F1 & $\mathbf{F 2}$ & $\mathbf{F} 3$ & $\mathbf{F}_{4}$ \\
\hline 08. Penso que estou saturado(a) pelo meu trabalho & & 0.38 & & \\
\hline 12. Sinto-me pressionado(a) pelo trabalho. & & 0.54 & & \\
\hline 17. Sinto-me cansado(a) fisicamente no trabalho & & 0.89 & & \\
\hline 18. Sinto-me desgastado(a) emocionalmente. & & 0.82 & & \\
\hline 02. Não gosto de atender alguns pacientes. & & & 0.45 & \\
\hline 03. Acho que muitos pacientes são insuportáveis & & & 0.60 & \\
\hline 06. Acho que os familiares dos pacientes são uns chatos & & & 0.64 & \\
\hline 07. Penso que trato com indiferença alguns pacientes. & & & 0.53 & \\
\hline 11. Gosto de ser irônico(a) com alguns pacientes & & & 0.56 & \\
\hline 14. Rotulo ou classifico os pacientes segundo o seu comportamento. & & & 0.50 & \\
\hline 04. Preocupa-me a forma como tratei algumas pessoas no trabalho & & & & 0.50 \\
\hline 09. Sinto-me culpado(a) por alguma das minhas atitudes no trabalho. & & & & 0.64 \\
\hline 13. Tenho remorsos por alguns dos meus comportamentos no trabalho. & & & & 0.64 \\
\hline $\begin{array}{l}\text { 16. Penso que deveria pedir desculpas a alguém pelo meu comportamen- } \\
\text { to no trabalho }\end{array}$ & & & & 0.84 \\
\hline 20. Sinto-me mal por algumas coisas que disse no trabalho. & & & & 0.86 \\
\hline Alfa de Crombach & 0.83 & 0.85 & 0.73 & 0.80 \\
\hline \% total de variância explicada & & & & \\
\hline
\end{tabular}

Os quatro fatores resultantes da AFE foram responsáveis por $64 \%$ da variação total e interpretados com base na perspectiva teórica, a saber:

Fator 1 (eigenvalues = 6,36), denominado "ilusão pelo trabalho", composto por cinco itens $(1,5,10$, $15,19)$ sendo o de menor carga fatorial $(0,69)$ o item 10 ("Penso que o meu trabalho me dá coisas positivas") e o de maior carga fatorial $(0,76)$ os itens 15 e 19 ("O meu trabalho me é gratificante" e "Sinto-me encantado(a) pelo meu trabalho").

Fator 2 (eigenvalues $=2,70$ ), denominado "desgaste psíquico", composto por quatro itens $(08,12,17,18)$ sendo o de menor carga fatorial $(0,38)$ o item 8 ("Penso que estou saturado(a) pelo meu trabalho") e o de maior carga fatorial $(0,89)$ o item 17 ("Sinto-me cansado(a) fisicamente no trabalho").
Fator 3 (eigenvalues $=1,51$ ), denominado "indolência", composto por seis itens $(2,3,6,7$. $11,14)$ sendo o de menor carga fatorial $(0,45) 0$ item 2 ("Não gosto de atender alguns pacientes") e o de maior carga fatorial $(0,64)$ o item 6 ("Acho que os familiares dos pacientes são uns chatos").

Fator 4 (eigenvalues = 1,21), denominado "culpa", composto por cinco itens $(4,9,13,16,20)$ sendo o de menor carga fatorial $(0,50)$ o item 4 ("Preocupa-me a forma como tratei algumas pessoas no trabalho") e o de maior carga fatorial $(0,86)$ o item 20 ("Sintome mal por algumas coisas que disse no trabalho").

Já com relação a análise de confiabilidade, todos os fatores apresentaram boa consistência interna, a saber: ilusão pelo trabalho $(\alpha)=0,83$; desgaste psíquico $(\alpha)=0,85$; indolência $(\alpha)=0,73$; e culpa $(\alpha)=0,80$. 


\section{Evidências de validade convergente}

Para obtenção de evidências de validade convergente da CQUEST com a JSS, foram realizadas correlações $r$ de Pearson, como apresentado na Tabela 4. Os resultados apresentaram-se teoricamente coerentes, indicando correlações pequenas e moderadas (Cohen, 1992) e significativas entre todos os fatores da CQUEST e da JSS.

Tabela 4 - Correlações entre os fatores da CQUEST e a JSS

JSS

CQUEST

\begin{tabular}{ccc}
\hline Controle & Demanda & Apoio Social \\
\hline $0.44^{* *}$ & $-0.19^{*}$ & $0.42^{* *}$ \\
$-0.30^{* *}$ & $0.34^{* *}$ & $-0.55^{* *}$ \\
$-0.23^{* *}$ & $0.19^{*}$ & $-0.32^{* *}$ \\
$-0.11^{*}$ & $0.26^{* *}$ & $-0.34^{* *}$ \\
\hline
\end{tabular}

${ }^{*} p<0,05 ;{ }^{* *} p<0.01$

\section{Discussão}

Em consonância com os resultados reportados, pode-se concluir que O CESQT apresenta evidências de validade de construto, em específico por meio da AFE e da convergência com outra variável, e indices de consistência interna aceitáveis para avaliação do Burnout em profissionais da saúde. Estes resultados indicam um modelo de quatro fatores, que se apresenta em consonância com os estudos de desenvolvimento do instrumento (Gil-Monte, 2005), da sua adaptação para o contexto brasileiro em uma amostra de 714 professores (Gil-Monte et al., 2010) e sua adaptação para outros paises, como o México (Gil-Monte \& Zúñiga-Caballero, 2010), Itália (Gil-Monte, Viotti, \& Converso, 2017) e Peru (Valverde, 2016).

Com relação aos resultados das evidências de validade convergente, foram apresentadas evidências que corroboram com o modelo proposto por Gil-Monte (2005). Tais evidências indicam que o instrumento apresenta uma relação teoricamente coerente com o estresse ocupacional. Em outras palavras, as evidências apontaram que profissionais da saúde com maiores escores na dimensão ilusão pelo trabalho são caracterizados por um elevado controle e apoio social no trabalho e uma baixa demanda; já elevados escores nas dimensões desgaste psiquico, indolência e culpa são característicos de pouco controle e pouco apoio social e uma elevada demanda. Esses resultados são coerentes não só com achados sobre Burnout em profissionais da saúde (Andolhe, Barbosa, Oliveira, Costa, \& Padilha, 2015; Vidotti, Ribeiro, Galdino, \& Martins, 2018), como em outros profissionais (ex. bancários (Coelho et al., 2018); policiais (Pelegrini, Cardoso, Claumann, Pinto, \& Felden, 2018). Assim, o CESQT providencia uma avaliação das dimensões relacionados ao Burnout, disponibilizando um instrumento com evidências de adequação psicométrica em profissionais da saúde, o que implica diretamente no processo de avaliação para formulação de estratégias de intervenções visando a redução do Burnout.

Mesmo atingindo o objetivo proposto, é importante indicar as possiveis limitações da presente pesquisa no intuito de que os resultados encontrados sejam compreendidos dentro do contexto ao qual foi realizada. Desse modo, acredita-se que, ainda que não tenha sido o objetivo da presente pesquisa, a utilização de uma amostragem não probabilística limita o poder de generalização dos resultados. Além disso, devese considerar as limitações inerentes ao tipo de instrumento utilizado (autorrelato), como é o caso 
da desejabilidade social. Ademais, aponta-se a não homogeneidade da amostra, composta técnicos de enfermagem, médicos, enfermeiros, odontólogos, fisioterapeutas, o que não deve ser entendido como uma limitação da pesquisa, mas como uma singularidade que deve ser observada diante dos resultados. Assim, estudos futuros poderão buscar evidências da adequação psicométrica (validade e fidedignidade) do CESQT em amostrar separadas especificamente pela profissão.

Outros aspectos, que podem ser explorados em estudos futuros, é a utilização do CESQT para verificar a associação do Burnout com variáveis que contribuem para o aumento dos niveis de estresse ocupacional, como duração do periodo de férias (Vasconcelos \& Martino, 2017), tempo de trabalho, tipo de vínculo (Vidotti et al., 2018). Compreendese também que diferentes tipos de evidência de validade devem ser verificados, como a busca de evidências relacionada ao critério, utilizando-se de amostras de trabalhadores afastados por conta do Burnout, ou evidências de validade divergente, por meio da avaliação da satisfação no trabalho (Souza, Milani, \& Alexandre, 2015). Recomendase ainda a realização de procedimentos de normatização em diferentes populações. Tais indicações, pesquisas futuras, devem contribuir para um melhor entendimento e refinamento psicométrico do CESQT e sua utilização para aferir os niveis de Burnout.

\section{Referências}

American Educational Research Association, American Psychological Association \& National Council on Measurement in Education. (2014). Standards for Educational and Psychological Testing ( $5^{\mathrm{a}}$ ed.). Washington, DC: American Educational Research Association.

Alves, M. G. M., Chor, D., Faerstein, E., Lopes, C. S., \& Werneck, G. L. (2004). Versão resumida da "job stress scale": adaptação para o português. Revista Saúde Pública, 38(2), 164-71. http://dx.doi.org/10.1590/S0034$\underline{89102004000200003}$

Andolhe, R., Barbosa, R. L., Oliveira, E. M., Costa, A. L. S., \& Padilha, K. G. (2015). Estresse, coping e burnout da Equipe de Enfermagem de Unidades de Terapia Intensiva: fatores associados. Revista da Escola de Enfermagem da USP, 49, 58-64. https://dx.doi.org/10.1590/ S0080-623420150000700009
Batista, J. B. V., Carlotto, M. S., Coutinho, A. S., \& Augusto, L. G. da S. (2011). Sindrome de Burnout: confronto entre o conhecimento médico e a realidade das fichas médicas. Psicologia em Estudo, 16(3), 429-435. http:// dx.doi.org/10.1590/S1413-73722011000300010

Bambula, F. D. \& Gómez, I. C. (2016). Pesquisa sobre a sindrome de burnout na América Latina entre 2000 e 2010. Psychology from the Caribbean, 33(1), 113-131. http://dx.doi.org/10.14482/psdc.33.1.8065

Benevides-Pereira, A. M. (2014). Burnout: O processo de adoecimento pelo trabalho. In A. M. Benevides-Pereira (Ed.), Burnout: Quando o trabalho ameaça o bem-estar do trabalhador (4ª ed., pp. 17-57). São Paulo, SP: Casa do Psicólogo.

Cardoso, H. F., Baptista, M. N., Sousa, D. F. A., \& Goulart Júnior, E. (2017). Sindrome de Burnout: análise da literatura nacional entre 2006 e 2015. Revista Psicologia Organizações e Trabalho, 17(2), 121-128. https://dx.doi. org/10.17652/rpot/2017.2.12796

Coelho, J. A. P. M., Souza, G. H. S., Cerqueira, C. L. C., Esteves, G. G. L., \& Barros, B. N. R. (2018). Estresse como preditor da Sindrome de Burnout em bancários. Revista Psicologia Organizações e Trabalho, 18(1), 306-315. https://dx.doi.org/10.176.52/rpot/2018.1.13162

Cohen, J. (1992). A power primer. Psychological Bulletin, $112,155-159$

Costa, L. S. T., Gil-Monte, P. R., Possobon, R. F., \& Ambrosano, G. M. B. (2013). Prevalência da Sindrome de Burnout em uma amostra de professores universitários brasileiros. Psicologia: Reflexão e Crítica, 26(4), 636-642. https://dx.doi.org/10.1590/S0102-79722013000400003

Ebling, M. \& Carlotto, M. S. (2012). Burnout syndrome and associated factors among health professionals of a public hospital. Trends in Psychiatry and Psychotherapy, 34(2). 93-100. https://doi.org/10.1590/S223760892012000200008

Gil-Monte P.R. (2005). El sindrome de quemarse por el trabajo (Burnout): una enfermedad laboral en la sociedad del bienestar. Madrid: Pirámide.

Gil-Monte, P. R., Carlotto, M. S., \& Câmara, S. G. (2010). Validação da versão brasileira do "Cuestionario para la Evaluación del Síndrome de Quemarse por el Trabajo" em professores. Revista de Saúde Pública, 44(1), 140-147. https://dx.doi.org/10.1590/S0034-89102010000100015

Gil-Monte, P. R., Viotti, S., \& Converso, D. (2017). Propiedades psicométricas del «Cuestionario para la Evaluación del Sindrome de Quemarse por el Trabajo» (CESQT) en profesionales de la salud italianos: una perspectiva de género. Liberabit, 23(2), 153-168. https://dx.doi.org/ https:// doi.org/10.24265/liberabit.2017.v23n2.01

Gil-Monte, P. R. \& Zúñiga-Caballero, L. C. (2010). Validez factorial del "Cuestionario para la Evaluación del Sindrome de Quemarse por el Trabajo" (CESQT) en una muestra de médicos mexicanos [Factorial validity of the "Spanish Burnout Inventory" (SBI) in a sample of Mexican doctors]. Universitas Psychologica, 9(1), 169-178. http://dx.doi.org/10.1037/t21442-000 
Gómez, S. H. M., Rivera, G. B. R., Tiznado, O. J., \& Grisales, V. D. (2018). Spanish Burnout inventory: A meta-analysis based approach. Contaduria y administración, 63(2). https://dx.doi.org/10.22201/fca.24488410e.2018.1120

Karasek, R., \& Theörell, T. (1990). Healthy work: Stress, productivity and the reconstruction of working life. New York: Basic Books.

Koga, G. K. C., Melanda, F. N., Santos, H. G., Sant'Anna, F. L., González, A. D., Mesas, A. E., \& Andrade, S. M. (2015). Fatores associados a piores níveis na escala de Burnout em professores da educação básica. Cadernos Saúde Coletiva, 23(3), 268-275. https://dx.doi.org/10.1590/ 1414-462X201500030121

Kristensen, T. S., Borrritz, M., Villadsen, E., \& Christensen, K. B. (2005). The Copenhagen Burnout Inventory: a new tool for the assessment of Burnout. Work Stress, 19(3),192207. https://dx.doi.org/10.1080/02678370500297720

Maslach, C., Schaufeli, W. B., \& Leiter, M. P. (2001). Job burnout. Annual Review of Psychology, 522(52), 397-422. https://doi.org/10.1146/annurev.psych.52.1.397

Ministério da Economia - Secretaria de Previdência (2020). Acompanhamento mensal do benefício auxílio-doença previdenciário concedido segundo os códigos da DID-10 - janeiro a dezembro de 2019. Recuperado de http://sa.previdencia.gov.br/site/2020/03/Acompanhamento-Mensal_Auxilio-Doenca-Previdenciario_2019_completo_CID-10.pdf

Moreira, H. A., Souza, K. N., \& Yamaguchi, M. U. (2018). Sindrome de Burnout em médicos: uma revisão sistemática. Revista Brasileira de Saúde Ocupacional, 43. https://dx.doi.org/10.1590/2317-6369000013316

Pelegrini, A., Cardoso, T. E., Claumann, G. S., Pinto, A. A., \& Felden, E. P. G. (2018). Percepção das condições de trabalho e estresse ocupacional em policiais civis e militares de unidades de operações especiais. Cadernos Brasileiros de Terapia Ocupacional, 26(2), 423-430. https://dx.doi.org/10.4322/2526-8910.ctoa01160

Poletto, N. A., Probst, L. F., Oliveira, T. L., Guerra, L. M., Ambrosano, G. M. B., Cortellazzi, K. L., Gil-Monte, P. R., \& Possobon, R. F. (2016). Sindrome de Burnout em gestores municipais da saúde. Cadernos de Saúde Coletiva, 24(2):209-215. https://dx.doi.org/10.1590/ 1414-462X201600020005

Silva, R. P., Barbosa, S. C., Silva, S. S., \& Patrício, D. F. (2015). Burnout e estratégias de enfrentamento em profissionais de enfermagem. Arquivos Brasileiros de Psicologia, 67(1), 130-145

Silveira, S. L. M., Câmara, S. G., \& Amazarray, M. R. (2014). Preditores da Sindrome de Burnout em profissionais da saúde na atenção básica de Porto Alegre/RS. Cadernos Saúde Coletiva, 22(4), 386-392. https://dx.doi. org/10.1590/1414-462X201400040012

Souza, A. C., Milani, D. \& Alexandre, N. M. C. (2015). Adaptação cultural de um instrumento para avaliar a satisfação no trabalho. Revista Brasileira de Saúde Ocupacional, 40(132), 219-227. https://dx.doi. org/10.1590/0303-7657000113715
Theorell, T. (1996). The demand-control-support model for studying health in relation to the work environment: an interactive model. In K. Orth-Gómer \& N. Schneiderman (Eds.), Behavioral medicine approaches to cardiovascular disease (pp. 69-85). Mahwah, NJ: Lawrence Erlbaum Associates

Trindade, L. L., Lautert, L., Beck, C. L. C., Amestoy, S. C., \& Pires, D. E. P. (2010). Estresse e sindrome de Burnout entre trabalhadores da equipe de Saúde da Familia. Acta Paulista de Enfermagem, 23(5), 684-689. https:// dx.doi.org/10.1590/S0103-21002010000500016

Valverde, Z. Y. V. (2019). Propiedades psicométricas del cuestionario para la evaluación del sindrome de quemarse por el trabajo en docentes del distrito de Juanjui, 2017 (Tese de doutorado não publicada, Faculdade de Humanas, Universidad César Vallejo) Tarapoto, Peru.

Vasconcelos, E. M. \& Martino, M. M. F. (2017). Preditores da sindrome de Burnout em enfermeiros de unidade de terapia intensiva. Revista Gaúcha de Enfermagem, 38(4), 128135. https://dx.doi.org/10.1590/1983-1447.2017.04.65354

Vidotti, V., Ribeiro, R. P., Galdino, M. J. Q., \& Martins, J. T. (2018). Burnout Syndrome and shift work among the nursing staff. Revista Latino-Americana de Enfermagem, 26, 250-264. https://dx.doi.org/10.1590/1518-8345.2550.3022

\section{Germano Gabriel Lima Esteves}

Doutorando em Psicologia Social do Trabalho e das Organizações pela Universidade de Brasilia (PSTO- UnB), em Brasilia, DF, Brasil; professor adjunto II da Universidade de Rio Verde (UniRV), em Rio Verde, GO, Brasil.

\section{Ana Adelaide Martins Leão}

Psicóloga pela Universidade de Rio Verde (UniRV), em Rio Verde, GO, Brasil.

\section{Esther de Oliveira Alves}

Psicóloga pela Universidade de Rio Verde (UniRV), em Rio Verde, GO, Brasil.

\section{Endereço para correspondência}

Germano Gabriel Lima Esteves

Universidade de Rio Verde/ Campus Rio Verde

Laboratório de Avaliação Psicológica de Rio Verde, s/n 75901-970

Rio Verde, Goiás, Brasil 